\title{
Psychosomatik und Osteopathie
}

\author{
Günter Steinfurth, Silvia Rudner
}

Immer wieder kommen Patienten in die osteopathische Praxis, deren Beschwerden keine erkennbare organische Ursache zugrunde zu liegen scheint. Meist bezeichnet man die Beschwerden als psychosomatisch. Doch was verbirgt sich genau hinter dieser Bezeichnung? Und ist in diesen Fällen die Osteopathie eine geeignete Behandlungsoption?

„Psychosomatose: Seele löst Krankheiten aus. Der Körper kann durch die Seele verrücktspielen. Schwindel, Atemnot, Kopfschmerzen oder Herzrasen können Anzeichen für psychische Belastungen sein. Psychosomatose heißt die Erkrankung, die durch die Seele ausgelöst wird und sich körperlich äußert. Die Betroffenen rennen oft von Arzt zu Arzt, ohne dass ihre Leiden anhaltend gemildert werden. Ihnen hilft letztlich eine Psychotherapie. Diese ist auch angebracht, wenn jemand z.B. unter Angst- und Panikattacken leidet." (Mitteldeutsche Zeitung: 14.11.2011). Der Begriff Psychosomatik oder ähnliche Wörter werden häufig und gerne als Schlagwort verwendet, wie auch in dieser kurzen Zeitungsnotiz. Sucht man jedoch nach einer einheitlichen Definition für „Psychosomatik“, wird man enttäuscht, denn nahezu jedes Lehrbuch weicht in der Formulierung mehr oder weniger vom anderen ab.

\section{Ein Begriff - viele Definitionen}

„Der Begriff ,Psychosomatische Medizin“ wird immer wieder missverstanden. Es handelt sich dabei zum einen um einen allgemeingültigen und selbstverständlichen Zugang zum notleidenden Menschen. Zum anderen ist es eine Fachdisziplin ... Zudem ist sie eine Forschungsrichtung mit dem Ziel, die seelische Seite einer

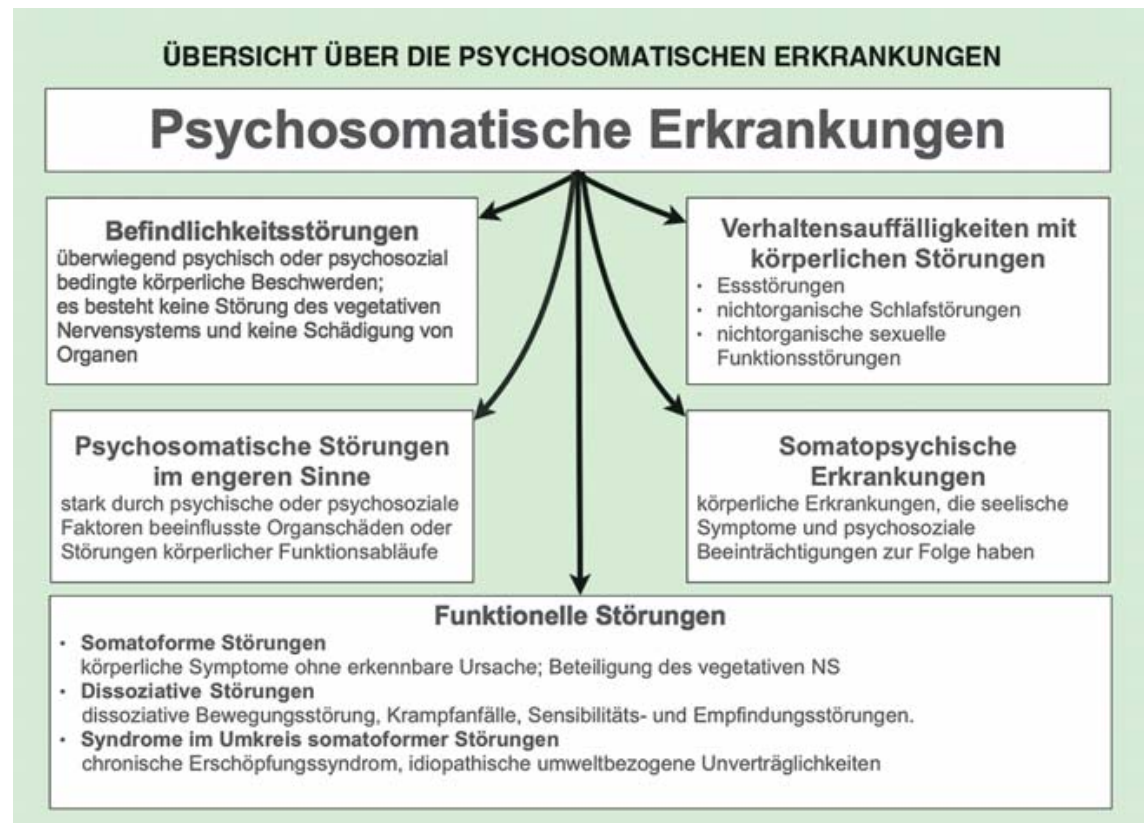

- Abb. 1 Übersicht über die psychosomatischen Erkrankungen.

Krankheit besser verstehen und damit besser behandeln zu können." [1]

In anderen Definitionen wird der „Zugang“ etwas näher erläutert: „Psychosomatische Medizin ist die Lehre von den körperlich-seelisch-sozialen Wechselwirkungen in der Entstehung, im Verlauf und in der Behandlung von menschlichen Krankheiten. Sie muss ihrem Wesen nach als eine personenzentrierte Medizin verstanden werden. “ [2] In der nachfolgenden Erklärung wird deutlich, dass bei psychosomatischen Erkrankungen auch seelische Anteile ausgemacht werden können. „Psychosomatik ist die Lehre von der Wechselwirkung zwischen seelischen, psychosozialen und körperlichen Prozessen in Gesundheit und Krankheit.“ [3]

Es geht also auch um Wechselwirkungen zwischen Körper, Seele und sozialem Umfeld. Man verlässt das vertraute Terrain von Ursache und Wirkung nicht. Das führt dazu, dass bestimmte Erkrankungen des
Körpers von der Seele ausgehen sollen, andere körperliche Erkrankungen auf die Seele wirken und bestimmte körperliche Symptome „rein psychisch“ sind. Glaubt man dann ein wenig Klarheit gefunden zu haben, raubt einem das folgende Zitat wieder alle Sicherheit: „Eine Unterscheidung zwischen psychosomatischen und rein somatischen Krankheiten ... kann weder theoretisch noch empirisch eingehalten werden." [6]

\section{Systematik der psycho- somatischen Erkrankungen}

In der medizinischen Krankheitslehre bestehen erhebliche Schwierigkeiten, Patienten mit körperlichen Symptomen ohne erkennbare organische Grundlagen ( $\triangleright$ Abb.1) zu beschreiben und einzuordnen. Es gibt dafür eine Fülle unterschiedlicher Bezeichnungen wie „psychovegeta- 
tives Syndrom“, „funktionelle Störung“, „vegetative Dystonie“, „psychogenes Syndrom“ oder „Organneurose“. Grundsätzlich spricht man von somatoformen Störungen, wenn keine organische Ursache für die Beschwerden gefunden werden kann, von psychosomatischen Störungen (im engeren Sinn) bei organischen Erkrankungen mit psychosozialen Auffälligkeiten.

Aktuell werden psychosomatische Erkrankungen klassifiziert als

- Befindlichkeitsstörungen,

- funktionelle Störungen,

- psychosomatische Störungen im engeren Sinn,

- Verhaltensauffälligkeiten mit körperlichen Störungen und

- somatopsychische Erkrankungen.

\section{Befindlichkeitsstörungen}

Man versteht darunter rein subjektive Empfindungen, die überwiegend psychisch oder psychosozial bedingt sind. Störungen des vegetativen Nervensystems und Schäden an Organen sind ausgeschlossen. Die häufigsten Befindlichkeitsstörungen sind Verdauungsstörungen, Kopfschmerzen, Schlafstörungen, aber auch z.B. Missstimmungen, Konzentrationsschwäche, Müdigkeit. Es finden sich aber auch Aussagen wie: „Befindlichkeitsstörungen gelten als typische Frauenleiden...“[7]
Auch wir kennen Patienten mit Befindlichkeitsstörungen aus der osteopathischen Praxis. Vielleicht neigen wir manchmal dazu, ihre Beschwerden nicht ganz ernst zu nehmen. Bei genauerer Untersuchung und gutem Hinhören finden wir mit den besonderen osteopathischen Diagnosemethoden Dysfunktionen, die wir erfolgreich behandeln können. Unbehandelt können diese Symptome mit der Zeit in funktionelle Störungen übergehen: „Wenig sinnvoll erscheint auch eine semantische Diskussion, ob noch eine Befindlichkeitsstörung oder bereits eine Erkrankung vorliegt, da die Übergänge fließend und pathophysiologisch nicht exakt definiert sind.“ [8]

\section{Funktionelle Störungen}

Bei den funktionellen Störungen unterscheidet man 3 Untergruppen:

- somatoforme Störungen

- dissoziative Störungen

- Syndrome im Umkreis somatoformer Störungen

\section{Somatoforme Störungen}

Somatoforme Störungen bezeichnen körperliche Symptome ohne erkennbare organische Ursache. Häufig unterstellt man eine Dysregulation des vegetativen Nervensystems. Sie können schon im Kindes- und Jugendalter auftreten. Die epidemiologische Verteilung kann aufgrund unzureichender Daten und der Diagnose-
Tab. 1 Kategorien der somatoformen Störungen nach ICD 10.

\begin{tabular}{|ll|}
\hline Kodierung & Art der somatoformen Störung \\
\hline F 45.0 & $\begin{array}{l}\text { Somatisierungsstörung } \\
\text { undifferenzierte Somatisie- } \\
\text { rungsstörung }\end{array}$ \\
\hline F 45.1 & $\begin{array}{l}\text { hypochondrische Störung } \\
\text { s } 45.2\end{array}$ \\
F 45.3 & $\begin{array}{l}\text { tionsstörung } \\
\text { anhaltende Schmerzstörung }\end{array}$ \\
F 45.4 & $\begin{array}{l}\text { sonstige somatoforme Störung } \\
\text { somatoforme Störung, nicht }\end{array}$ \\
F 45.8 & näher bezeichnet \\
\hline
\end{tabular}

kriterien nur geschätzt werden [9]. Somatoforme Störungen sind nach den Phobien die häufigste psychische Störung. Ihre Prävalenz liegt bei Erwachsen bei 11-31\% und bei Kindern und Jugendlichen bei etwa $10 \%$.

Somatoforme Störungen werden in 7 weitere Gruppen unterteilt ( $\triangleright$ Tab. 1).

- Die Symptome der Somatisierungsstörung können sich auf jeden Teil des Körpers oder jedes System des Körpers beziehen. Sie reichen von Kopf- und Gesichtsschmerzen über Thorax- und Oberbauchschmerzen sowie Schmerzen des Bewegungsapparats und des Unterbauchs bis zu Ermüdung und Erschöpfung, Schwindel und Übelkeit. Der Verlauf der Störung ist chronisch und fluktuierend. Ist die Symptomatik weniger auffallend und dauert sie noch 
keine 2 Jahre an, spricht man von der undifferenzierten Somatisierungsstörung.

- Bei der hypochondrischen Störung steht die Beschäftigung mit der Krankheit oder die Möglichkeit, schwer erkrankt zu sein, im Vordergrund.

- Somatoforme autonome Funktionsstörungen zeigen häufig das Bild einer vegetativen Dysregulation eines bestimmten Organsystems. Dazu zählen die Herzneurose, die Magenneurose, das Colon irritabile (Reizdarm), die Hyperventilation und Dyspnoe sowie Dysurie und Polyurie. Studien haben schon mehrfach den positiven Einfluss der osteopathischen Behandlung bei diesen Störungen gezeigt [10-14].

- Bei der anhaltenden Schmerzstörung leiden die Patienten über mehrere Monate an Schmerzen in einer oder mehreren Körperregionen. Es können keine körperliche Ursachen gefunden werden, welche die Beschwerden erklären. Oft klagen die Patienten auch über Erschöpfungszustände und vegetative Begleiterscheinungen, z. B. Herzphänomene, Verdauungsbeschwerden, Schwitzen. „Die Diagnose somatoforme Schmerzstörung (ICD-10: F45.4) wird häufig erst nach mehrjähriger Krankheitsdauer und multiplen diagnostischen Abklärungen, teilweise auch iatrogenen Schädigungen gestellt. “ [15] Unter iatrogenen Schäden verstehen wir auch eine wenig einfühlsame, verstörende, den seelischen Konflikt noch verstärkende Sprache und Haltung des Arztes oder Therapeuten.

- Sonstige somatoforme Störungen sind Störungen der Wahrnehmung, der Körperfunktion und des Krankheitsverhaltens, die nicht durch das vegetative Nervensystem vermittelt werden, die auf spezifische Teile oder Systeme des Körpers begrenzt sind und mit belastenden Ereignissen oder Problemen eng in Verbindung stehen, wie Dysmenorrhö, Dysphagie, einschließlich „Globus hystericus“, Pruritus, Tortikollis, Bruxismus. Da wir diese Beschwerden aus der osteopathischen Praxis als Indikationen gut kennen, muss hier gut eruiert werden, ob ein deutlicher $\mathrm{Zu}$ sammenhang mit belastenden Ereignissen oder Problemen besteht; es gilt, einfühlsam vorzugehen und evtl. den

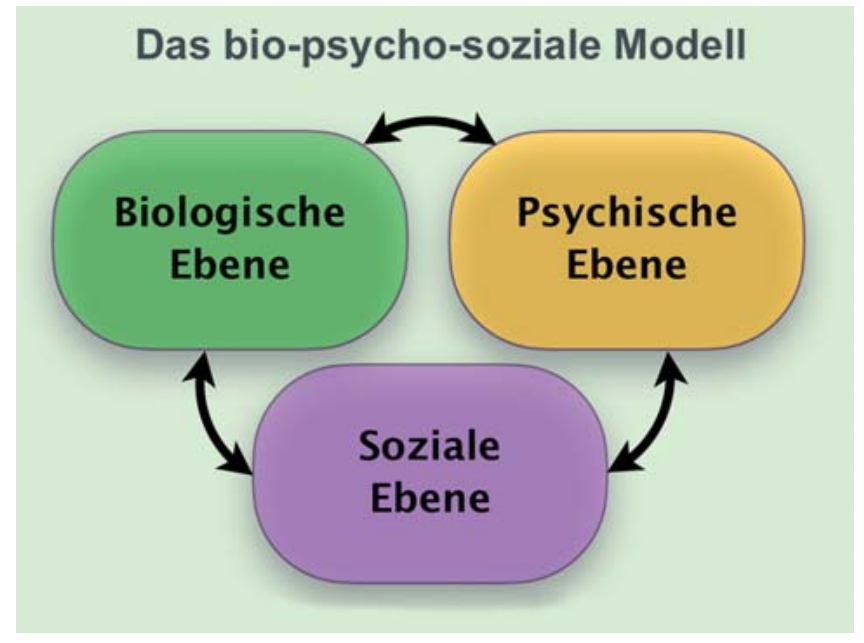

Abb. 2 Das biopsychosoziale Modell.

Patient für eine (zusätzliche) Psychotherapie zu sensibilisieren.

Unserer Erfahrung nach sollte gerade in diesen Bereichen die Zusammenarbeit mit Psychotherapeuten gesucht werden.

\section{Dissoziative Störungen}

Durch traumatisierende Erlebnisse, unerträgliche Konflikte oder gestörte Beziehungen kann es bei manchen Menschen zu sog. dissoziativen Störungen (Konversionsstörungen in der Psychoanalyse) kommen. Das heißt: Psychische und körperliche Funktionen sind entkoppelt. Man unterscheidet kognitiv-dissoziative Störungen (z.B. psychogene Gedächtnisstörung) und körperlich-dissoziative Störungen. Letztere können sich als Bewegungsstörungen, Krampfanfälle oder Sensibilitätstörungen äußern.

\section{Syndrome im Umkreis somatoformer Störungen}

Syndrome, deren Ätiologie nicht gesichert ist, werden teilweise noch zu den somatoformen Störungen gezählt. Dazu gehören z.B. chronischer Tinnitus, idiopathische umweltbezogene Unverträglichkeiten, Fibromyalgie [16], das chronische Erschöpfungssyndrom. Die aktuelle Forschung zeigt allerdings, dass hier oft deutliche physische Veränderungen zu finden sind [17].

\section{Psychosomatische Störungen im engeren Sinne}

Hier handelt es sich um Organschäden oder Störungen physiologischer Funktionen, bei denen psychische oder soziale Aspekte ausschlaggebend für die Entste- hung, Aufrechterhaltung oder Verschlimmerung der Erkrankung sind. Bei der Diagnose ist es daher wichtig, beide Faktoren der Krankheit zu berücksichtigen - d.h. den Menschen als Ganzes zu sehen. Diese Definition nach der ICD-10 bricht mit der althergebrachten Beschreibung von Franz Gabriel Alexander, der 1950 eine erste Liste von 7 psychosomatischen Erkrankungen erstellte, die später als „Holy Seven“ bezeichnet wurden.

1. Ulcus ventriculi und Ulcus duodeni

2. Asthma bronchiale

3. Rheumatoide Arthritis

4. Neurodermitis

5. Essenzielle Hypertonie

6. Hyperthyreose

7. Colitis ulcerosa, Morbus Crohn

Er verband mit den jeweiligen Erkrankungen bestimmte Persönlichkeitstypen. Diese (psychoanalytische) Sichtweise, der viele Therapeuten unterschiedlicher Fachrichtungen noch folgen, ist im Hinblick auf Forschungen im Bereich der biologischen Psychologie nicht mehr aktuell und - wie der Name „Holy Seven“ schon andeutet eher eine Glaubensfrage. Wenn man Patienten, die an diesen Erkrankungen leiden, von vornherein einen psychischen Hintergrund unterstellt, wie es diese „Definition“ nahelegt, läuft man Gefahr, sie zu stigmatisieren. Heute geht man bei den Psychosomatosen, die auch als Organneurosen oder vegetative Neurosen bezeichnet werden, von einem multifaktoriellen Entstehungskonzept aus. 


\section{Verhaltensauffälligkeiten mit körperlichen Störungen}

Zu den Verhaltensauffälligkeiten mit körperlichen Störungen gehören nach der ICD-10 v.a. Essstörungen, nichtorganische Schlafstörungen und die nichtorganischen sexuellen Funktionsstörungen. Nach dieser Auffassung können Änderungen des Verhaltens oder der seelischen Verfassung auch körperliche Erkrankungen auslösen oder verschlimmern.

\section{Somatopsychische Erkrankungen}

Somatopsychische Erkrankungen bezeichnen die psychischen und sozialen Folgeerscheinungen organischer Krankheiten, z.B. Krebs, Aids, Diabetes mellitus, Schilddrüsenerkrankungen, rheumatische Erkrankungen, Epilepsie oder chronische Niereninsuffizienz.

\section{Psychosomatik - neu gedacht}

Schon 1977 stellte G.L. Engel in Science sein biopsychosoziales Modell ( $\triangleright$ Abb. 2) als Alternative zum biomedizinischen Modell vor [18]. Er geht davon aus, dass jede Krankheit eine körperliche, psychische und soziale Komponente hat. Erkrankung kann und sollte unter diesen 3 Aspekten betrachtet, analysiert, erforscht und behandelt werden. Nach diesen Erkenntnissen klingt dann auch die folgende Definition der Psychosomatik viel befriedigender und zukunftsweisender: „Unter Psychosomatik wird eine Betrachtungsweise von Gesundheit und Krankheit in der Medizin verstanden, die den Menschen als eine einzigartige geistig-seelisch-sozial-körperliche Einheit versteht. Sie betont beim Vorliegen von körperlichen Störungen die Berücksichtigung von geistig-seelischen und sozialen Faktoren für das Verständnis von Krankheitsentwicklung und Heilung.“ [19]

\section{Fazit}

Muss es bei so vielen Wechselwirkungen zwischen Psyche und Soma nicht heißen: Psychotherapie für Osteopathen und Hands on für Psychotherapeuten? Ganz klar: Nein! Schuster bleib bei deinen Leisten! Das Fachwissen und die erforderlichen Kenntnisse und Fertigkeiten übersteigen bei Weitem die Möglichkeiten des Einzelnen.

Die Osteopathie kann bei vielen der o.g. Störungen einen wichtigen Beitrag leisten. Dabei muss der Osteopath emphatisch auf seinen Patienten eingehen und sehr sorgfältig erwägen, ob weitere diagnostische oder therapeutische Maßnahmen erforderlich und welche osteopathischen Maßnahmen angebracht sind.

In unserer Praxis haben wir die Erfahrung gemacht: Die Arbeit des einen verbessert die Erfolge des anderen, ein Aus-

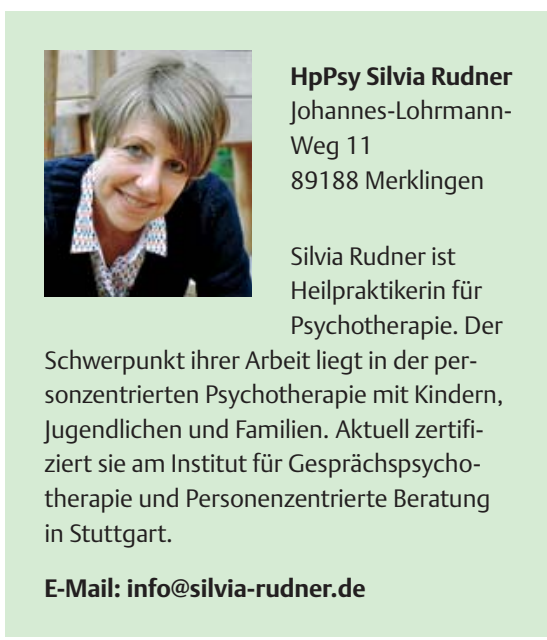

Günter Steinfurth D. O., M. R.O FSCCO HP Johannes-LohrmannWeg 11 89188 Merklingen Günter Steinfurth graduierte 1995 an der SKOM. Er unterrichtet regelmäßig bei der Salutaris Akademie vorwiegend „Osteopathie im kranialen Bereich“ und „Einführung in die osteopathische Pädiatrie“. Seit 2014 betreibt er zusammen mit Frau Rudner eine interdisziplinäre Praxis.

E-Mail: E-Mail: Msteinfurt@aol.com 